\title{
Pricing European Option When the Stock Price Process Is Being Driven by Geometric Brownian Motion
}

\author{
Kebareng I. Moalosi-Court \\ Department of Mathematics and Statistical Sciences, Botswana International University of Science and Technology, Palapye, \\ Botswana \\ Email: kebarengc@gmail.com
}

How to cite this paper: Moalosi-Court, K.I. (2019) Pricing European Option When the Stock Price Process Is Being Driven by Geometric Brownian Motion. Open Access Library Journal, 6: e5568.

https://doi.org/10.4236/oalib.1105568

Received: July 1, 2019

Accepted: July 28, 2019

Published: August 2, 2019

Copyright $\odot 2019$ by author(s) and Open Access Library Inc.

This work is licensed under the Creative Commons Attribution International License (CC BY 4.0).

http://creativecommons.org/licenses/by/4.0/

\section{(c) (i) Open Access}

\begin{abstract}
This report is about modeling a European Option in general when the stock price process is being driven by geometric Brownian motion $(\mathrm{gBm})$. The volatility parameter is used as an example of a basic estimator and simulated values of geometric Brownian motion hence exploring some of the properties that improve the accuracy of an estimator. The theory is then extended to estimate the volatility from real data by using the Roger-Satchell Estimator. Hence the estimated volatility is used in the model developed in calculating the value on European option using the Donsker Delta Function approach and is compared with that of the Black-Scholes formula. A unique finding is an observation that the value of an option obtained from using the Donsker Delta Function approach is more of the European Put Option than European Call Option which uses the Black-Scholes formula, then this roughly leads to the conclusion that the Donsker Delta Function approach computes a European Put Option.
\end{abstract}

\section{Subject Areas \\ Mathematical Economics \\ Keywords \\ Option Pricing, Donsker Delta function, Black-Scholes and Geometric Brownian Motion}

\section{Introduction}

We model European option when the stock price is driven by the geometric Brownian motion $(\mathrm{gBm})$, and one should note that the $\mathrm{gBm}$ has wide acceptance 
as a standard model for the growth of a price of a stock over time. Also literature indicates that option pricing theory was first developed by R.C. Merton, F. Black and M.S. Scholes in the early 1970s. Based on the no-arbitrage principle, they derived a theoretical valuation formula for European Call and Put on a $\mathrm{gBm}$ driven stock price (see Brandt [1], Korn [2] and Magdon-Ismail [3]).

Mitra [4] stated that in the pricing of the option the volatility parameter plays a vital role; it helps us understand price dynamics since it is one of the key variables in a stochastic differential equation governing an asset pricing. As such in this paper we developed a formula or volatility estimator; its purpose is to explore some of the properties that improve the accuracy of an estimator and also give insight into the roots of the estimator to finding the volatility from real data. This was achieved by calibration of the volatility parameter from two sets of simulated data of the $\mathrm{gBm}$. Using the theory developed, the volatility parameter is then estimated from real data using the Roger-Satchell [5] volatility estimator. Hence the estimated volatility was used in calculating the value on European option using the Donsker Delta Function approach the model developed by Aase [6] and the Black-Scholes formula, then comparison of the option value on Donsker Delta Function approach of option pricing and on the Black-Scholes formula was done. There is a wide literature on pricing of European option, so the reader can further see these related works; Flint [7] in their paper estimated long-term volatility for market models and Rouah [8] priced European Option and the volatility parameter using excel-VBA (Visual Basic for Applications).

The paper is organised as follows: Section 2 gives the model layout of the paper. The next section addresses the concept of Donsker Delta Function and its application in Option Pricing [6]. Section 4 explores ways of estimating the volatility parameter from historical data. Section 5 deals with the actual pricing of an option, then finally conclusion section.

\section{The Model}

We consider our market model as defined whereby we have a risk and risk-less asset. We also consider that we are in a complete Black-Scholes market whereby the dynamics of our asset prices is modeled by the following two securities

1) A risk free asset, where $X_{0}(t)$ per unit time is given by

$$
\mathrm{d} X_{0}(t)=\rho(t) X_{0}(t) \mathrm{d} t ; X_{0}(0)=1
$$

2) A risky asset, where $X_{1}(t)$ per unit time is given by

$$
\mathrm{d} X_{1}(t)=\mu(t) X_{1}(t) \mathrm{d} t+\sigma(t) X_{1}(t) \mathrm{d} B t ; X_{1}(0)=x>0 ;
$$

$\rho(t), \mu(t)$ and $\sigma(t)$ are deterministic functions with the property

$$
\int_{0}^{T}\left(|\rho(s)|+|\mu(s)|+\sigma^{2}(s)\right) \mathrm{d} s<\infty
$$

satisfied by Itô's Lemma.

Similar to [6] we assume that $\sigma$ is bounded away from zero. This condition is to ensure that the stochastic term is not eliminated, hence otherwise it be- 
comes a deterministic function. $\mathbb{P}$ will denote the probability measure of $B(t)$ and $\mathcal{F}_{t}$ denote the $\sigma$-algebra generated by $\left\{B_{s}(.)\right\}_{s \leq t}$.

Now let $\phi:[0, T] \rightarrow \mathbb{R}$ be another deterministic function such that $\int_{0}^{T} \phi^{2}(t) \mathrm{d} t<\infty$ and

$$
Z(t)=\int_{0}^{t} \phi(s) \mathrm{d} B(s) ; 0 \leq t \leq T
$$

Let $F$ be the contingent claim of the memory-less type, i.e. it is Markovian. It is given by

$$
F=h(Z(T))
$$

where $h: \mathbb{R} \rightarrow \mathbb{R}$ is a bounded measurable function.

We let $\left(\theta_{0}(t), \theta_{i}(t)\right), i=1,2, \cdots, n$ be the portfolio and $\theta_{0}(t), \theta_{1}(t)$ are $\mathcal{F}_{t}$-adapted stochastic process referred as the number of stock held by an investor at time $t$. Then

$$
V(t)=\theta_{0}(t) X_{0}(t)+\theta_{1}(t) X_{1}(t)
$$

is defined the value of this portfolios and the change in the value process is known as self-financing. This means no external funds are topped up to the portfolio. From Equation (5)

$$
\theta_{0}(t)=\frac{V(t)-\theta_{1}(t) X_{1}(t)}{X_{0}(t)}
$$

Then substituting into (5) and using (1) and (2), we get

$$
\begin{aligned}
\mathrm{d} V(t) & =\left(V(t)-\theta_{1}(t) X_{1}(t)\right) \frac{\mathrm{d} X_{0}(t)}{X_{0}(t)}+\theta_{1}(t) \mathrm{d} X_{1}(t) \\
& =\left(V(t)-\theta_{1}(t) X_{1}(t)\right) \rho \mathrm{d} t+\theta_{1}(t) \mu(t) X_{1}(t) \mathrm{d} t+\theta_{1}(t) \sigma(t) X_{1}(t) \mathrm{d} B(t) \\
& =\rho(t) V(t) \mathrm{d} t+\theta_{1}(t) X_{1}(t)[(\mu(t)-\rho(t)) \mathrm{d} t+\sigma(t) \mathrm{d} B(t)]
\end{aligned}
$$

since $\sigma(t) \neq 0$ for a.a.t, then we let $\alpha(t)=\frac{\mu(t)-\rho(t)}{\sigma(t)}$ to get

$$
\mathrm{d} V(t)=\rho(t) V(t) \mathrm{d} t+\theta_{1}(t) \sigma(t) X_{1}(t)[\alpha \mathrm{d} t+\mathrm{d} B(t)]
$$

from (7) we get the integrating factor

$$
\begin{gathered}
\int_{0}^{t} \frac{\mathrm{d} V(s)}{V s}=\int_{0}^{t} \rho(s) \mathrm{d} s \\
V(t)=\mathrm{e}^{\int_{0}^{t} \rho(s) \mathrm{d} s}
\end{gathered}
$$

We then multiply (7) by an integrating factor becomes

$$
\mathrm{d}\left(\mathrm{e}^{-\int_{0}^{t} \rho(s) \mathrm{d} s} V(t)\right)=\mathrm{e}^{-\int_{0}^{t} \rho(s) \mathrm{d} s} \theta_{1}(t) \sigma(t) X_{1}(t)[\alpha \mathrm{d} t+\mathrm{d} B(t)] .
$$

Therefore integrating the above equation leads to

$$
\mathrm{e}^{-\int_{0}^{T} \rho(s) \mathrm{d} s} V(T)=V(0)+\int_{0}^{T} \mathrm{e}^{-\int_{0}^{t} \rho(s) \mathrm{d} s} \theta_{1}(t) \sigma(t) X_{1}(t)[\alpha \mathrm{d} t+\mathrm{d} B(t)]
$$

Suppose the value process $\{V(t), 0 \leq t \leq T\}$ has 


$$
V(T)=F
$$

at time $t=T$ and $t=0$ it has the market value $V(0)$. We also require that the value process be of an admissible portfolio. By combining (8) with (6) we see that it suffices to find $V(0)$ and a process $u(t, \omega)$ such that

$$
\mathrm{e}^{-\int_{0}^{T} \rho(s) \mathrm{d} s} F=V(0)+\int_{0}^{T} \mu(t)[\alpha(t) \mathrm{d} t+\mathrm{d} B(t)]
$$

and

$$
\mathbb{P}\left[\int_{0}^{T} \mu^{2}(s) \mathrm{d} s<\infty\right]=\mathbb{P}\left[\int_{0}^{T}|\mu(s) \alpha(s)| \mathrm{d} s<\infty\right]=1
$$

such that $\left\{\int_{0}^{T} \mu(t)[\alpha \mathrm{d} t+\mathrm{d} B(t)]\right\}_{t \in[0, T]}$ is lower bounded, and if a process such as $\mu(t)$ exist, then

$$
\theta_{1}(t)=\mathrm{e}^{\int_{0}^{t} \rho(s) \mathrm{d} s} \sigma_{t}^{-1} X_{1}^{-1} u(t, \omega)
$$

and solve for $\theta_{0}(t)$ using (6). Then by the Girsanov theorem it is known that if

$$
\int_{0}^{t} \alpha^{2}(s) \mathrm{d} s<\infty,
$$

therefore $\mathrm{V}(0)$ is unique and given by

$$
V(0)=\mathbb{E}_{\mathbb{Q}}\left[\mathrm{e}^{-\int_{0}^{t} \rho(s) \mathrm{d} s} F\right]
$$

where the expectation is with respect to the measure $\mathbb{Q}$ define on $\mathcal{F}_{T}$ to make the process to be a martingale. The next section deals with Donsker Delta function, in which the first main theorems are generalized with white noise function.

\section{The Donsker Delta Function}

Definition 1. Consider $(\mathcal{S})^{*}$, the set of Hida distribution function. Suppose we have a random variable $X: \Omega \rightarrow \mathbb{R}$ such that $X \in(\mathcal{S})^{*}$. The Donsker delta function of $X$ is the function

$$
\delta_{X}(.): \mathbb{R} \rightarrow(\mathcal{S})^{*}
$$

with the property that

$$
\int_{\mathbb{R}} f(x) \delta_{X}(x) \mathrm{d} x=f(X), \text { a.s }
$$

for all (measurable) $f: \mathbb{R} \rightarrow \mathbb{R}$ for which the integral converges.

This is one of the distribution parameters in space $(\mathcal{S})^{*}$ (for more information see Aase and colleagues [6]).

Proposition 3.1. Consider a normally distributed random variable $X$ with mean $m$ and variance $v>0$. Then $\delta_{Y}$ is unique and is given by the expression

$$
\delta_{X}(x)=\frac{1}{\sqrt{2 \pi v}} \cdot \exp ^{\diamond}\left[-\frac{\left(x-X_{1}(T)\right)^{\diamond 2}}{2 v}\right] \in(\mathcal{S})^{*} .
$$

See Aase, et al. [6] for proof. 
According to the above Proposition, the Donsker Delta is just a normal distribution function of random variable $X$.

Lemma 3.2. Let $\mu:[0, T] \rightarrow \mathbb{R}, \sigma:[0, T] \rightarrow \mathbb{R}$ be deterministic functions and such that $\int_{0}^{T}|\mu(s)| \mathrm{d} s<\infty$ and $\|\sigma\|_{[0, T]}^{2}=\int_{0}^{T} \sigma^{2}(s) \mathrm{d} s<\infty$. Define

$$
X_{1}(t)=\int_{0}^{t} \mu(s) \mathrm{d} s+\int_{0}^{t} \sigma(s) \mathrm{d} B_{s}, 0 \leq t \leq T .
$$

Then

$$
\begin{aligned}
& \exp ^{\diamond}\left[-\frac{\left(x-X_{1}(t)\right)^{\diamond 2}}{2\|\sigma\|_{[0, T]}^{2}}\right]=\exp ^{\diamond}\left[-\frac{x^{2}}{2\|\sigma\|_{[0, T]}^{2}}\right] \\
& +\int_{0}^{T} \exp ^{\diamond}\left[-\frac{\left(x-X_{1}(t)\right)^{\diamond 2}}{2\|\sigma\|_{[0, T]}^{2}}\right] \diamond \frac{x-X_{1}(t)}{2\|\sigma\|_{[0, T]}^{2}} \diamond\left(\mu(t)+\sigma(t) B_{t}\right) \mathrm{d} t .
\end{aligned}
$$

Observe, in Equation (16) in the lemma says is that if $X_{1}(t)$ is a gBm, then Equation (17) holds. The above proposition and lemma provide the pre-request needed for the development of theorem we are interested in. [refer to Aase and colleagues [6] for proofs].

Theorem 3.3. Suppose we have two deterministic functions $\sigma:[0, T] \rightarrow \mathbb{R}$, $\alpha:[0, T] \rightarrow \mathbb{R} \quad$ which are square integrable, i.e.;

$$
0<\|\sigma\|_{[0, T]}^{2}:=\int_{0}^{T} \sigma^{2}(s) \mathrm{d} s<\infty \text { and } 0 \leq \int_{0}^{T} \alpha^{2}(s) \mathrm{d} s<\infty .
$$

Let

$$
X_{1}(t)=\int_{0}^{t} \sigma(s) \mathrm{d} B_{s}+\int_{0}^{t} \sigma(s) \alpha(s) \mathrm{d} s ; 0 \leq t \leq T
$$

and $f: \mathbb{R} \rightarrow \mathbb{R}$ be bounded. Then

$$
f\left(X_{1}(T)\right)=V_{0}+\int_{0}^{T} u(t, w) \diamond\left(\alpha(t)+W_{t}\right) \mathrm{d} t,
$$

where

$$
V_{0}=\int_{\mathbb{R}} \frac{f(x)}{\sqrt{2 \pi}\|\sigma\|_{[0, T]}} \exp \left[-\frac{x^{2}}{2\|\sigma\|_{[0, T]}^{2}}\right] \mathrm{d} x
$$

and

$$
u(t)=\sigma(t) \cdot \int_{\mathbb{R}} \frac{f(x)}{\sqrt{2 \pi}\|\sigma\|_{[0, T]}} \exp ^{\diamond}\left[-\frac{\left(x-X_{1}(T)\right)^{\diamond 2}}{2\|\sigma\|_{[0, T]}^{2}}\right] \diamond\left[-\frac{x-X_{1}(T)}{\|\sigma\|_{[0, T]}^{2}}\right] \mathrm{d} x .
$$

Also see Aase and colleagues [6] for proof, and note that $\mu(s)=\sigma(s) \alpha(s)$. Equation (20) shows the value of the claim which is dependent to the value of $X_{1}$ at $t=T$, and the function $\mathrm{f}$ is bounded so that the portfolio be admissible. The formula (20), (21) and (22) provide us with the way of calculating the value of an option, the initial investment value $\left(V_{0}\right)$ of an option and the portfolio respectively. However the formula is not user friendly, as such needs to be specified. 
The above theorem was then transferred into a non-Wick version and gave a more familiar representation in the theorem that follows.

Theorem 3.4. Suppose $\sigma(t), X_{1}(t)$ be as in Theorem (3.3) and assume that

$$
\|\sigma\|_{[t, T]}^{2}:=\int_{t}^{T} \sigma^{2}(s) \mathrm{d} s>0, \text { for all } t<T,
$$

and $f: \mathbb{R} \rightarrow \mathbb{R}$ be bounded. Then

$$
f\left(X_{1}(T)\right)=V_{0}+\int_{0}^{T} \mu(t, \omega)\left(\alpha(t)+W_{t}\right) \mathrm{d} t,
$$

and where

$$
V_{0}=\int_{\mathbb{R}} \frac{f(x)}{\sqrt{2 \pi}\|\sigma\|_{[0, T]}} \exp \left[-\frac{x^{2}}{2\|\sigma\|_{[0, T]}^{2}}\right] \mathrm{d} x
$$

and

$$
u(t)=\sigma(t) \cdot \int_{\mathbb{R}} \frac{f(x)}{\sqrt{2 \pi}\|\sigma\|_{[0, T]}} \exp \left[-\frac{\left(x-X_{1}(T)\right)^{2}}{2\|\sigma\|_{[0, T]}^{2}}\right]\left[-\frac{x-X_{1}(T)}{\|\sigma\|_{[0, T]}^{2}}\right] \mathrm{d} x
$$

\section{Calibration of the Volatility}

The process of option pricing involves the volatility parameter $\sigma$ and in modeling of the stock price, volatility is used as one of the parameters in the Black-Scholes formula. To obtain or estimate this parameter historical data is used. Never-the-less several methods have been proposed on how $\sigma$ can be estimated (see Flint [7], Hurn [8], Rouach and Vainberg [9]). It is indispensable that before embarking actual pricing of options, we find ways of calculating this parameter. So the goal of this section is to explore ways of estimating the volatility parameter from the data.

\subsection{Estimation of Volatility Parameter}

Consider the gBm process as modeled by Equation (2). This means that at any time interval $[t, T]$

$$
\ln \left(X_{1}(T)\right)-\ln \left(X_{1}(t)\right)=\ln \left(\frac{X_{1}(T)}{X_{1}(t)}\right) \sim N\left[\left(\mu-\frac{\sigma^{2}}{2}\right)(T-t), \sigma^{2}(T-t)\right],
$$

then considering a very small time interval $[t-1, t]$ and letting $\Delta t$ to be a small change in time, then $Y_{t}$ can be written as

$$
Y_{t}=\ln \left(X_{1}(t)\right)-\ln \left(X_{1}(t-1)\right)=\ln \left(\frac{X_{1}(t)}{X_{1}(t-1)}\right)
$$

and it follows that

$$
Y_{t} \sim N\left[\left(\mu-\frac{\sigma^{2}}{2}\right) \Delta t, \sigma^{2} \Delta t\right]
$$

from which 


$$
\begin{gathered}
\mathbb{E}\left(Y_{t}\right)=\left(\mu-\frac{\sigma^{2}}{2}\right) \Delta t \\
\operatorname{Var}\left(Y_{t}\right)=\sigma^{2} \Delta t .
\end{gathered}
$$

How then does one estimate the sample variance? From the general theory of statistics let the sample mean $\bar{Y}$ be

$$
\bar{Y}=\frac{\sum_{t=1}^{n} Y(t)}{n}, n>0
$$

hence the sample variance is

$$
s_{Y}=\sqrt{\frac{1}{n-1} \sum_{t=1}^{n}(Y(t)-\bar{Y})^{2}} .
$$

Therefore, the estimate for the sample variance will be

$$
\hat{\sigma}=\frac{s_{Y}}{\Delta t} .
$$

As shown from the formulae developed, this estimation is achieved by discretizing the process. In other words though the process is a continuous process, the estimation is achieved by using some values of the process. For the development of this concept, we then immediately concentrate on calibrating the volatility parameter from the simulated values of $\mathrm{gBm}$.

The 99 simulated values of the $\mathrm{gBm}$ is shown in the Appendix section, Table $\mathrm{B} 1$. These values are treated as artificial data in the calibration of volatility parameter. Having simulated the $\mathrm{gBm}$ using the known drift and volatility parameters, we now try to use our method on the values of $\mathrm{gBm}$ to retrieve back the $\sigma$. For programming Equation (30) we use Matlab (see program (1) in Appendix), and using it on the 99 simulated values of $\mathrm{gBm}$ from Table B1 in column titled $\Delta t=0.0002$. The estimated variance gives $\hat{\sigma}=0.2151$. Similarly using values generated when $\Delta t=0.0004$, the estimated variance is $\hat{\sigma}=0.2216$. Note that $\Delta t=0.0004$ sampled only 49 simulated values.

Figure 1 was created to observe the trend of the estimated volatility, and Figure 2 then shows the error between the estimated and actual values of sigma. This was done using the formula Error $=|\hat{\sigma}-\sigma|$.

Note in graphs since the time values are very small numbers the estimates were plotted against the number of samples for convenience sake. But it should also be taken note that as time increases the number of samples also increases. Figure 1 are graphs of the values of estimates of the volatility parameter as the time increases. The purple graph is the graph of volatility estimates generated using the simulated values of $\mathrm{gBm}$ when the interval is $\Delta t=0.0002$ (Appendix B, Table B1 and column 1). Similarly red graph was generated when the values of $\mathrm{gBm}$ were simulated with interval $\Delta t=0.0004$ (Appendix B, Table B1 and column 2). Taking into account that the real value of the volatility is $\sigma=0.23$ (all these values were simulated using this volatility), the graphs shows that the values of the volatility estimates generated using values simulated when $\Delta t=0.0004$ gives a better estimates than using the values generated when $\Delta t=0.002$. 


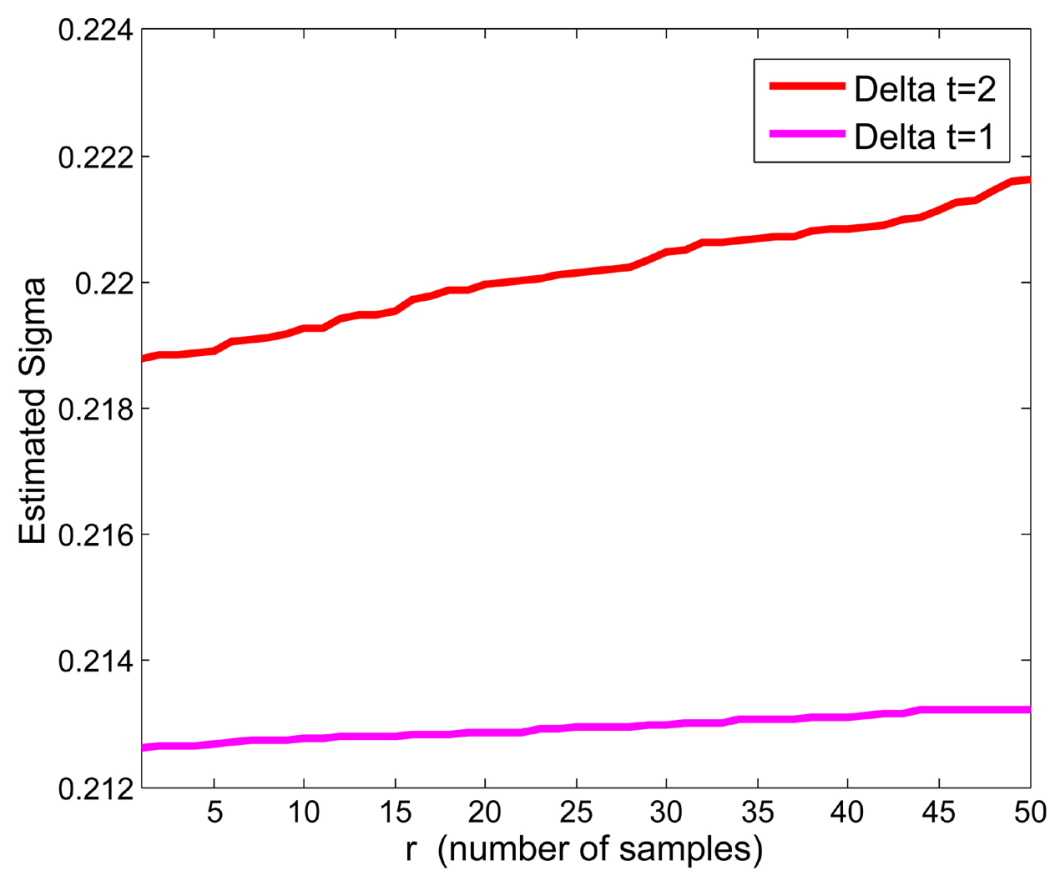

Figure 1. Estimated sigma from the simulated values of $\mathrm{gBm}$ using $\Delta t=0.0002$ and $\Delta t=0.0004$.

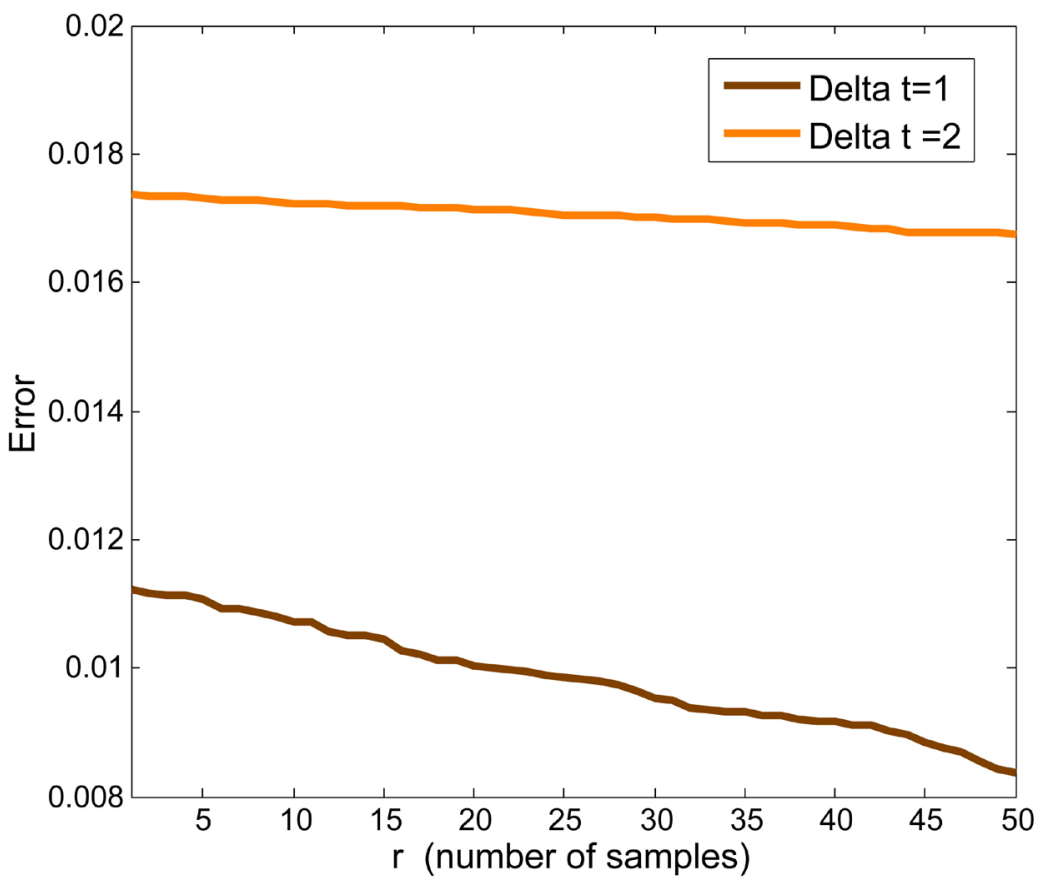

Figure 2. Error difference between the estimated and actual sigma.

Figure 2 gives the graphs of the error difference between the estimated and actual value of volatility estimates as the time increases. From this figure we observe that the orange graph of error between the volatility estimates is generated when using the values of $\mathrm{gBm}$ values simulated when $\Delta t=0.0002$, while brown graph are the errors generated when using of $\mathrm{gBm}$ values simulated when 
$\Delta t=0.0004$. Hence from the results we observe that $\Delta t=0.0004$ gives a better estimates than those values generated when using values of gBm generated when $\Delta t=0.0002$ as the values of error graph of the former gives lower values than those of the latter. We also observe that both error graphs decreases as time increases.

These results show that the length of time interval between successive values has an influence in the accuracy of the estimates of the volatility parameter. It can also be concluded that the sample size has influence on the accuracy of the estimator. As observed from Figure 2 the error size decreases as time and sample size increases, this means we can get more accurate estimates if we use a large sample size of values of $\mathrm{gBm}$ than using a small sample size. These are some of the results which may affect the accuracy of our estimates. Some of our findings can be confirmed by the findings of [3]. The other factor which may also affect the efficiency of an estimator which [3] also mention is that of the $\mu \geq 0$, which they discussed in their paper.

\subsection{Calibration Volatility Parameters from Real Data}

We then develop our theory further to calibrating the volatility parameter to actual data, however there are some departures in using the gBm and the actual asset price process. One of the major departure is the existence of open jumps, rather than being continuous. Most asset markets are closed overnight, weekends and for certain holidays, as such information arriving during the periods when the market is closed often results in open prices being different from the closing prices.

The estimator (Equation (30)) might not be efficient when applied to real data because of these departures, but its purpose was to explore some of the properties that improve the accuracy of an estimator and also give insight of the roots of the estimators which are used to estimate volatility from real data. There are a number of other estimators which are more efficient. For example some of the well known estimators are Parkinson, Garman and Klass, Roger and Satchell, Alizahdeh, Brandt and Diebold, and Yang and Zhang they use information on daily trading range, refer to a paper by [1]. For this paper we use the Roger-Satchell estimator, it uses the open, high, low and open prices, as such we assume that the close price of any given day is the open of the next day. [10] denote high and low by $h$ and $I$ respectively, i.e.,

$$
\begin{aligned}
& h=\sup _{0 \leq t \leq T} X_{1}(t), \\
& l=\inf _{0 \leq t \leq T} X_{1}(t),
\end{aligned}
$$

where $T$ is the period of the length. Hence, the estimate is given by

$$
\hat{\sigma}_{R S}=\sqrt{\frac{1}{N T} \sum_{i=1}^{N}\left(h_{i}-o_{i}\right)\left(h_{i}-c_{i}\right)+\left(l_{i}-o_{i}\right)\left(l_{i}-c_{i}\right)}
$$

where $N$ is the total number of the sample and $T$ is the period of the length. The 
real data on stock prices that is used in this research was obtained from the Dow Jones close, high and low values from $04 / 01 / 10$ to $13 / 12 / 10$. This represents a total of 240 days. The time interval of a day was considered, i.e. $\Delta t=0.0002$. Then the volatility was retrieved by using the Roger-Satchell estimator (33). A Matlab program (see program (1) in Appendix) for this estimator was developed to attain the volatility, which is

$$
\hat{\sigma}_{R S}=0.057630 \text {. }
$$

As mentioned above the Dow Jones stock indices were used. One should know that stock indices represent relative change in the share/stock price of constituent companies of shares that make up the index. Stock indices have several uses like the provision of history of a market movements level, providing a tool of estimating future movement of a market based on the past trend. There are several types of indices like the total return indices, the unweighed arithmetic indices, weighed arithmetic indices and the geometric indices just to mention a few. There are several examples of indices and one of the common examples and one used in this research is the Dow Jones Stock Indices. This is an unweighed arithmetic index that provide industrial factor.

\section{Calculating the Value of an Option}

We then use Theorem (3.4), Equation (25) to find the value of an option in real stock prices. Practically this is first achieved by calculating the implied volatility

$$
\rho=\frac{\ln [1+r]}{T}
$$

where $r$ is the interest rate of the stock price. The volatility implied by the market price can be determined by inverting the option pricing formula, [11]. In our paper the implied volatility assist in the calculation of the initial value of the integral of Equation (25), which is

$$
x=\ln \left[\mathrm{e}^{-\rho T} \frac{K}{X_{1}(0)}\right]+\frac{1}{2} \sigma T,
$$

where $K$ is the strike price, $X_{1}(0)$ the initial price and $f(x)$ is defined as

$$
f(x)=\mathrm{e}^{-\rho T} \frac{K}{X_{1}(T)}\left(\mathrm{e}^{x-X_{1}(T)}-1\right) .
$$

Hence $V_{0}$ can be simplified as

$$
V_{0}=\int_{x}^{T} \frac{f(x)}{\sqrt{2 \pi T \sigma^{2}}} \exp \left[-\frac{x^{2}}{2 T \sigma^{2}}\right] \mathrm{d} x .
$$

We therefore used the above simplified formulas (35) and (36) to model Equation (37) in Matlab, in our program we used the $\hat{\sigma}_{R S}=0.057630$, and let $X_{1}(0)$ be the first open price. The rate of interest we used was $r=0.07$, we choose $K=3964.20$ and through our calculation we obtained the value of an option using the Donsker Delta Function approach as 


$$
V_{0}=325.3121 \text {. }
$$

Comparison was made between this calculated value of an option using the Donsker Delta Function approach and the value of the European Call and Put options by using the Black-Scholes formula. The Black-Scholes formula is the earliest for calculating the European options. This option pricing theory was developed by R.C. Merton, F. Black and M.S. Scholes in the early 1970s. Based on the no-arbitrage principle, they derived a theoretical valuation formula for European Call and Put on a gBm driven stock price. The European Call option can be calculated by the formula,

$$
C=X_{1}(0) \Phi(\omega)-K \exp ^{-r T} \Phi(\omega-\sigma \sqrt{t})
$$

while the European Put option is given by

$$
P=K \exp ^{-r T} \Phi(-\omega+\sigma \sqrt{t})-X_{1}(0) \Phi(-\omega)
$$

where

$$
\omega=\frac{r t+\frac{\sigma^{2} t}{2}-\ln \left(\frac{K}{X_{1}(0)}\right)}{\sigma \sqrt{t}},
$$

$X_{1}(0)$ and $K$ are as defined before, $r$ is the interest rate, $\sigma$ is the volatility and $\Phi(\omega)$ is the normal distribution function. From Statistics and theory of integrals, the integral can be decomposed as

$$
\Phi(\omega)=\frac{1}{\sqrt{\pi}} \int_{-\infty}^{\frac{\omega}{\sqrt{2}}} \mathrm{e}^{-t^{2}} \mathrm{~d} t=\frac{1}{\sqrt{\pi}}\left(\int_{-\infty}^{0} \mathrm{e}^{-t^{2}} \mathrm{~d} t+\frac{1}{\sqrt{\pi}} \int_{0}^{\frac{\omega}{\sqrt{2}}} \mathrm{e}^{-t^{2}} \mathrm{~d} t\right) .
$$

Using the theory of improper integrals

$$
\frac{1}{\sqrt{\pi}} \int_{-\infty}^{\infty} \mathrm{e}^{-t^{2}} \mathrm{~d} t=\sqrt{\pi}
$$

hence

$$
\frac{1}{\sqrt{\pi}} \int_{-\infty}^{0} \mathrm{e}^{-t^{2}} \mathrm{~d} t=\frac{\sqrt{\pi}}{2}
$$

and

$$
\Phi(\omega)=\frac{1}{2}\left\{1+\operatorname{erf}\left(\frac{\omega}{\sqrt{2}}\right)\right\},
$$

where erf is the error function, defined by

$$
\operatorname{erf}(\omega)=\frac{2}{\sqrt{\pi}} \int_{0}^{\omega} \mathrm{e}^{-t^{2}} \mathrm{~d} t .
$$

[5] state that this error function is similar to distribution function of the standard normal distribution.

In programming the European Call and Put in Matlab, Equation (41) was taken into consideration in simulating the Normal distribution function. It should be noted that Matlab already have the in-built error function, that calcu- 
late the error function given by Equation (42). A complete program (1) of the European Call and Put is given in the Appendix section and it yielded the following results

$$
\text { Call }=8.3912
$$

and

$$
\text { Put }=225.9648 \text {. }
$$

Table 1 below was constructed to give a better observation of the results we obtained above by varying the initial value of the stock. The program for giving the results in Table 1, was a combination of program (1) and (1), and it was designed such that the $t, r, \sigma$ and $K$ were kept constant.

From Table 1, the following observations were made; the Donsker delta approach represented by $V_{0}$ gives a higher price of an option as compared to Black-Scholes formula, the values of $V_{0}$ are more close to that of the Put option than the Call option, the values of the Put option are greater than of the Put option price, also as $X_{i}(0)$ increases it was noted that the value of $V_{0}$ and Call option increases, and as $X_{i}(0)$ increases Put option decreases.

Never-the-less, the practical use of the Donsker Delta Function approach $V_{0}$ compared to the Black-Scholes formula gave the above results and observations. We justify this differences looking at the design and implications in this formulas; it can be concluded that the developed value of the $V_{0}$ gives more of the Put than the Call option, and the difference between the value of $V_{0}$ and Put option may be brought by some parameters in the formulas. e.g Looking at the $V_{0}$ formula, it uses the implied volatility given by Equation (35).

\section{Conclusion}

In this paper we first we estimate the volatility parameter and we observe that the length of interval between two successive values and the sample size has an

\begin{tabular}{|c|c|c|c|c|}
\hline \multicolumn{4}{|c|}{ Donsker Delta Approach } & \multirow{2}{*}{$\begin{array}{c}\text { Black-Scholes Formulae } \\
\text { Put }\end{array}$} \\
\hline$i$ & Initial Price $X_{i}(0)$ & $V_{0}$ & Call & \\
\hline 0 & 3568.3 & 325.3121 & 8.3744 & 225.9578 \\
\hline 1 & 3569.3 & 325.5422 & 8.4817 & 225.0651 \\
\hline 2 & 3570.3 & 325.7711 & 8.5896 & 224.1784 \\
\hline 3 & 3571.4 & 326.0221 & 8.7090 & 223.2079 \\
\hline 4 & 3572.3 & 326.2317 & 8.8098 & 222.3987 \\
\hline 5 & 3573.3 & 326.4621 & 8.9216 & 221.5105 \\
\hline 6 & 3574.3 & 326.6938 & 9.0352 & 220.6185 \\
\hline 7 & 3575.0 & 326.8516 & 9.1132 & 220.0120 \\
\hline 8 & 3576.3 & 327.1560 & 9.2651 & 218.8440 \\
\hline 9 & 3577.3 & 327.3856 & 9.3810 & 217.9644 \\
\hline
\end{tabular}

Table 1. Comparison. 
effect on the accuracy of an estimator. We also calculate the value of the European option using the Donsker Delta Function approach and the Black-Scholes formula, although they have some differences in the values; we found that the Donsker Delta Function approach of pricing an option gives more of the European Put Option than European Call Option. The reader can further extend this work using the Donsker Delta Function approach to find the value of the portfolio at any time $t$, by using Equation (26). According to Hafner [12], practically at anytime $t$, this can be achieved by finding the stock price at that day which is usually quoted in newspapers and put

$$
X_{t}=\ln \left[\mathrm{e}^{-\rho(T-t)} \frac{K}{X_{1}(0)}\right]+\frac{1}{2} \sigma(T-t),
$$

and

$$
u(t)=\int_{x-X_{1}(t)}^{\infty} \frac{f(x)}{\sqrt{2 \pi \sigma^{2}(T-t)}} \exp \left[-\frac{\left(x-X_{1}(t)\right)^{2}}{2 \sigma^{2}(T-t)}\right]\left[-\frac{x-X_{1}(t)}{T-t}\right] \mathrm{d} x .
$$

Now using the calculated $V_{0}$ and this portfolio, calculations can be made to find out if really the claim is replicated. Similarly using the Black-Scholes formula approach, the value of the portfolio can be calculated by using the formula

$$
\theta(t, \omega)=\Phi\left[\sigma^{-1}(T-t)^{-\frac{1}{2}}\left(\ln \frac{X_{1}(t)}{K}+\rho(T-t)+\frac{1}{2} \sigma^{2}(T-t)\right)\right]
$$

by Øksendal [13], then comparison can be made about these values of the portfolios.

\section{Acknowledgements}

My great thanks goes to Dr Elias Offen for his useful comments.

\section{Conflicts of Interest}

The author declares no conflicts of interest regarding the publication of this paper.

\section{References}

[1] Brandt, W.M. and Kinlay, J. (2005) Estimating Historical Volatility. http://www.investment-analytics.com

[2] Korn, E. and Korn, R. (2001) Option Pricing and Portfolio Optimization: Model Methods of Financial Mathematics. American Mathematical Society, Providence. https://doi.org/10.1090/gsm/031

[3] Magdon-Ismail, M. and Atiya, A.F. (2001) A Maximum Likelihood Approach to Estimating for a Brownian Motion Using the High, Low and Closing. Quantitative Finance, 1, 19.

[4] Mitra, S. (2009) Review on Volatility and Option Pricing.

[5] Rogers, L. and Setchell, S. (1994) Estimating Variance from High, Low and Closing Prices. Annals of Applied Probability, 1, 504-512. 
https://doi.org/10.1214/aoap/1177005835

[6] Aase, K., Øksendal, B. and Ubøe, J. (1998) Using the Donker Delta Function to Compute Hedging Strategies. Potential Analysis, 14, 359-360.

[7] Flint, E.J., Ochse, E. and Polokow, D.A. (2014) Estimating Long-Term Volatility Parameters for Market Consistent Models. South African Actuarial Journal, 14, 19-72. https://doi.org/10.4314/saaj.v14i1.2

[8] Hurn, A.S., Lindsay, K.A. and McClelland, J. (2012) Estimating the Parameters of Stochastic Volatility Models Using Option Price Data. Working Paper.

[9] Rouah, F.D. and Vainberg, G. (2007) Option Pricing Models and Volatility Using Excel-VBA. Wiley, Hoboken.

[10] Liang, Y.-Y. (2003) Demand Modeling with the Geometric Brownian Motion Process. Technical Report 1, T001.

[11] Merton, R.C. (1969) Life Portfolio Selection of Uncertainty: The Continuous Time Case. Review of Economic and Statistics, 51, 247-257. https://doi.org/10.2307/1926560

[12] Hafner, R. (2004) Stochastic Implied Volatility: A Factor-Based Model. Springer, Berlin. https://doi.org/10.1007/978-3-642-17117-8

[13] Oksendal, B. (2003) Stochastic Differential Equations: An Introduction with Application. Springer, Berlin. https://doi.org/10.1007/978-3-642-14394-6_5

[14] Ugur, O. (2008) Introduction to Computational Finance. Imperical College Press, London. https://doi.org/10.1142/p556

[15] Cox, J.C. and Ross, S.A. (1976) The Valuation of Options for Alternative Stochastic Processes. Journal of Financial Economics, 3, 145-166.

https://doi.org/10.1016/0304-405X(76)90023-4 


\section{Appendix A}

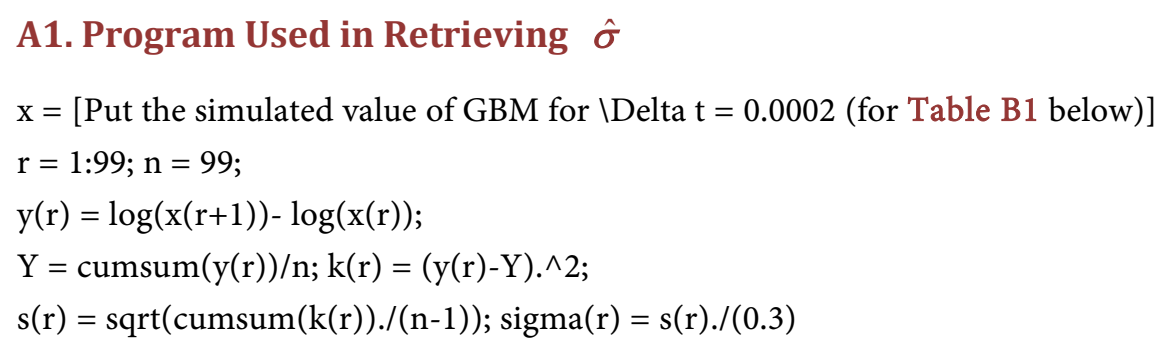

A2. Program for Retrieving the Volatility Using the Roger-Satchell Estimator

$\mathrm{h}=\left[\mathrm{DJC} \_\right.$High $]$

$1=[$ DJC_Low $]$

$\mathrm{o}=\mathrm{c}=$ [DJC_Open]

$\mathrm{N}=240$;

$\mathrm{r}=1: 240$;

$\mathrm{T}=240$;

$\mathrm{d}(\mathrm{r})=\left((\mathrm{h}(\mathrm{r})-\mathrm{o}(\mathrm{r})) .^{*}(\mathrm{~h}(\mathrm{r})-\mathrm{c}(\mathrm{r}))\right)+\left((\mathrm{l}(\mathrm{r})-\mathrm{o}(\mathrm{r})) .^{*}(\mathrm{l}(\mathrm{r})-\mathrm{c}(\mathrm{r}))\right)$

$\mathrm{h}(\mathrm{r})=\operatorname{cumsum}(\mathrm{d}(\mathrm{r})) \operatorname{sgrs}=\operatorname{sqrt}\left(\left(1 /\left(\mathrm{N}^{\star} \mathrm{T}\right)\right)^{\star} \mathrm{h}(\mathrm{r})\right)$

A3. Program for Retrieving the Donsker Delta Method for the Value of an Option Using $\hat{\sigma}_{R S}=\mathbf{0 . 0 5 7 6 3 0}$

$\mathrm{h}=$ [Put the DJC_High values from Table B2 below $]$

$1=$ [Put the DJC_Low values from Table B2 below $]$

$\mathrm{o}=\mathrm{c}=$ [Put the DJC_Open values from Table B2 below $]$

$\mathrm{N}=240 ; \mathrm{r}=1: \mathrm{N} ; \mathrm{T}=\mathrm{N} ;$ sgrs $=0.0576$;

syms $\mathrm{x}$

$\mathrm{X} 0=\mathrm{o}(1) ; \mathrm{K}=3964.20$;

$\mathrm{mu}=0.5 ; \mathrm{k}=0.07 ; \mathrm{v}=\log (1+\mathrm{k}) / \mathrm{T}$;

$\mathrm{g}=\exp \left(-\mathrm{v}^{\star} \mathrm{T}\right)^{\star}(\mathrm{K} / \mathrm{X} 0)$;

$\mathrm{x} 0=\log (\mathrm{g})+(1 / 2)^{\star}\left(\text { sgrs. }{ }^{\wedge} 2\right)^{\star} \mathrm{T} ;$

$\mathrm{fx}=\exp \left(-\mathrm{v}^{\star} \mathrm{T}\right)^{\star} \mathrm{K} .{ }^{\star}(\exp (\mathrm{x}-\mathrm{x} 0)-1)$;

$\mathrm{l}=\mathrm{fx} .{ }^{*} \exp \left(-\left(\mathrm{x}^{\wedge} 2\right)\right)$;

$\mathrm{c}=1 . /\left(2^{\star} \mathrm{T} .{ }^{*} \operatorname{sgrs} . \wedge 2\right)$;

$\mathrm{b}=\operatorname{sqrt}\left(2^{\star}\right.$ pi $^{\star} \mathrm{T} .{ }^{*}$ sgrs. $\left.{ }^{\wedge} 2\right)$;

$\mathrm{V}=\operatorname{double}(\operatorname{int}((\mathrm{c} . / \mathrm{b}), \mathrm{x}, \mathrm{x} 0, \mathrm{~T}))$

A4. Program for Retrieving the European Call and Put Option Using $\hat{\sigma}_{R S}=\mathbf{0 . 0 5 7 6 3 0}$

$\mathrm{h}=$ [Put the DJC_High values from Table B2 below $]$

$1=$ [Put the DJC_Low values from Table B2 below]

$\mathrm{o}=\mathrm{c}=$ [Put the DJC_Open values from Table B2 below]

$\mathrm{r}=1: 240 ; \mathrm{t}=240 / 365 ; \mathrm{R}=0.07 ; \mathrm{sgm}=0.0576$;

$\mathrm{K}=3964.20 ; \mathrm{X} 0=\mathrm{o}(1)$; 


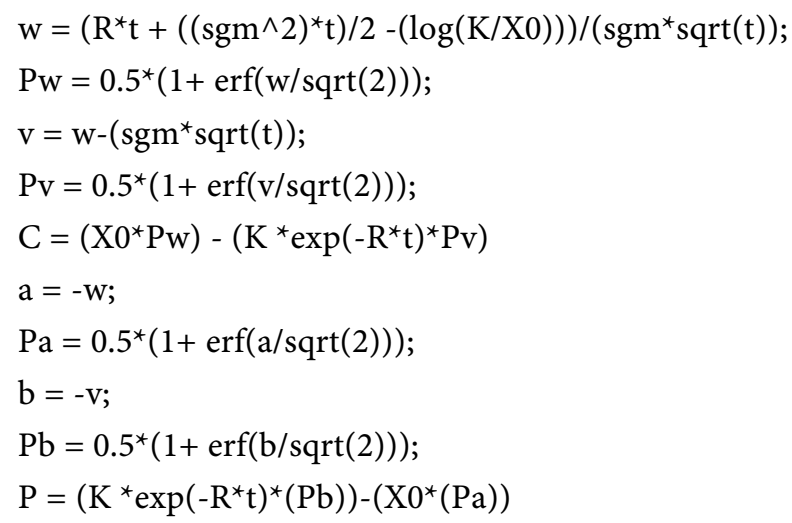

\section{Appendix B}

Table B1. Simulated values of geometric brownian motion for $\Delta t=0.0002$ and $\Delta t=0.0004$.

\begin{tabular}{|c|c|c|c|c|c|}
\hline GBM & $\Delta t=0.0002$ & $\Delta t=0.0004$ & GBM & $\Delta t=0.0002$ & $\Delta t=0.0004$ \\
\hline $\operatorname{gbm}(0)$ & 1 & 1 & $\operatorname{gbm}(50)$ & 1.9452 & 1.5878 \\
\hline $\operatorname{gbm}(1)$ & 1.8986 & & $\operatorname{gbm}(51)$ & 1.9491 & \\
\hline $\operatorname{gbm}(2)$ & 1.892 & 1.5981 & $\operatorname{gbm}(52)$ & 1.9433 & 1.5873 \\
\hline $\operatorname{gbm}(3)$ & 1.8994 & & $\operatorname{gbm}(53)$ & 1.9446 & \\
\hline $\operatorname{gbm}(4)$ & 1.911 & 1.5972 & $\operatorname{gbm}(54)$ & 1.9494 & 1.5917 \\
\hline $\operatorname{gbm}(5)$ & 1.904 & & $\operatorname{gbm}(55)$ & 1.949 & \\
\hline $\operatorname{gbm}(6)$ & 1.8963 & 1.6007 & $\operatorname{gbm}(56)$ & 1.9466 & 1.5917 \\
\hline $\operatorname{gbm}(7)$ & 1.8978 & & $\operatorname{gbm}(57)$ & 1.95 & \\
\hline $\operatorname{gbm}(8)$ & 1.9022 & 1.6073 & $\operatorname{gbm}(58)$ & 1.9461 & 1.5823 \\
\hline $\operatorname{gbm}(9)$ & 1.897 & & $\operatorname{gbm}(59)$ & 1.941 & \\
\hline $\operatorname{gbm}(10)$ & 1.8944 & 1.6081 & $\operatorname{gbm}(60)$ & 1.9404 & 1.5748 \\
\hline $\operatorname{gbm}(11)$ & 1.8937 & & $\operatorname{gbm}(61)$ & 1.9506 & \\
\hline $\operatorname{gbm}(12)$ & 1.8968 & 1.5956 & $\operatorname{gbm}(62)$ & 1.9511 & 1.5762 \\
\hline $\operatorname{gbm}(13)$ & 1.8942 & & $\operatorname{gbm}(63)$ & 1.9479 & \\
\hline $\operatorname{gbm}(14)$ & 1.9067 & 1.6012 & $\operatorname{gbm}(64)$ & 1.9406 & 1.5658 \\
\hline $\operatorname{gbm}(15)$ & 1.9072 & & $\operatorname{gbm}(65)$ & 1.9379 & \\
\hline $\operatorname{gbm}(16)$ & 1.9098 & 1.6017 & $\operatorname{gbm}(66)$ & 1.9394 & 1.571 \\
\hline $\operatorname{gbm}(17)$ & 1.9079 & & $\operatorname{gbm}(67)$ & 1.9426 & \\
\hline gbm(18) & 1.92 & 1.6006 & $\operatorname{gbm}(68)$ & 1.9483 & 1.5739 \\
\hline $\operatorname{gbm}(19)$ & 1.9172 & & $\operatorname{gbm}(69)$ & 1.9518 & \\
\hline $\operatorname{gbm}(20)$ & 1.9181 & 1.5949 & $\operatorname{gbm}(70)$ & 1.9626 & 1.5785 \\
\hline $\operatorname{gbm}(21)$ & 1.9299 & & $\operatorname{gbm}(71)$ & 1.9515 & \\
\hline $\operatorname{gbm}(22)$ & 1.9301 & 1.6025 & $\operatorname{gbm}(72)$ & 1.9435 & 1.5794 \\
\hline $\operatorname{gbm}(23)$ & 1.9193 & & $\operatorname{gbm}(73)$ & 1.9363 & \\
\hline $\operatorname{gbm}(24)$ & 1.9169 & 1.5897 & $\operatorname{gbm}(74)$ & 1.9365 & 1.5875 \\
\hline
\end{tabular}




\section{Continued}

\begin{tabular}{|c|c|c|c|c|c|}
\hline $\operatorname{gbm}(25)$ & 1.9107 & & $\operatorname{gbm}(75)$ & 1.9236 & \\
\hline $\operatorname{gbm}(26)$ & 1.915 & 1.5896 & $\operatorname{gbm}(76)$ & 1.9348 & 1.5829 \\
\hline $\operatorname{gbm}(27)$ & 1.9151 & & $\operatorname{gbm}(77)$ & 1.9349 & \\
\hline $\operatorname{gbm}(28)$ & 1.9245 & 1.5964 & $\operatorname{gbm}(78)$ & 1.9276 & 1.5867 \\
\hline $\operatorname{gbm}(29)$ & 1.9224 & & $\operatorname{gbm}(79)$ & 1.9262 & \\
\hline $\operatorname{gbm}(30)$ & 1.9284 & 1.5934 & $\operatorname{gbm}(80)$ & 1.9364 & 1.5961 \\
\hline $\operatorname{gbm}(31)$ & 1.9263 & & $\operatorname{gbm}(81)$ & 1.9366 & \\
\hline $\operatorname{gbm}(32)$ & 1.9276 & 1.5797 & $\operatorname{gbm}(82)$ & 1.9377 & 1.5966 \\
\hline $\operatorname{gbm}(33)$ & 1.9337 & & $\operatorname{gbm}(83)$ & 1.9419 & \\
\hline $\operatorname{gbm}(34)$ & 1.9209 & 1.5772 & $\operatorname{gbm}(84)$ & 1.9445 & 1.6019 \\
\hline $\operatorname{gbm}(35)$ & 1.9237 & & $\operatorname{gbm}(85)$ & 1.9433 & \\
\hline $\operatorname{gbm}(36)$ & 1.9198 & 1.5694 & $\operatorname{gbm}(86)$ & 1.934 & 1.5972 \\
\hline $\operatorname{gbm}(37)$ & 1.9299 & & $\operatorname{gbm}(87)$ & 1.9437 & \\
\hline $\operatorname{gbm}(38)$ & 1.9319 & 1.5824 & $\operatorname{gbm}(88)$ & 1.9417 & 1.5971 \\
\hline $\operatorname{gbm}(39)$ & 1.9348 & & $\operatorname{gbm}(89)$ & 1.952 & \\
\hline $\operatorname{gbm}(40)$ & 1.9327 & 1.5775 & $\operatorname{gbm}(90)$ & 1.9566 & 1.5861 \\
\hline $\operatorname{gbm}(41)$ & 1.9244 & & $\operatorname{gbm}(91)$ & 1.9544 & \\
\hline $\operatorname{gbm}(42)$ & 1.9215 & 1.5762 & $\operatorname{gbm}(92)$ & 1.9464 & 1.5785 \\
\hline $\operatorname{gbm}(43)$ & 1.921 & & $\operatorname{gbm}(93)$ & 1.9419 & \\
\hline $\operatorname{gbm}(44)$ & 1.9109 & 1.5806 & $\operatorname{gbm}(94)$ & 1.9428 & 1.5772 \\
\hline $\operatorname{gbm}(45)$ & 1.9148 & & $\operatorname{gbm}(95)$ & 1.9411 & \\
\hline $\operatorname{gbm}(46)$ & 1.9166 & 1.5821 & $\operatorname{gbm}(96)$ & 1.9431 & 1.5654 \\
\hline $\operatorname{gbm}(47)$ & 1.9255 & & $\operatorname{gbm}(97)$ & 1.9358 & \\
\hline $\operatorname{gbm}(48)$ & 1.9353 & 1.5788 & $\operatorname{gbm}(98)$ & 1.9359 & 1.5535 \\
\hline $\operatorname{gbm}(49)$ & 1.9436 & & $\operatorname{gbm}(99)$ & 1.9234 & \\
\hline
\end{tabular}

Table B2. Price values for the 2010 stock, for the Dow Jones index.

\begin{tabular}{cccc}
\hline DJC_th_High & DJC_th_Open & DJC_th_Low & Date \\
\hline 3631.60439 & 3568.315527 & 3588.781943 & $1 / 4 / 2010$ \\
3638.391043 & 3563.312034 & 3602.727848 & $1 / 5 / 2010$ \\
3638.577343 & 3577.218019 & 3600.186181 & $1 / 6 / 2010$ \\
3626.933576 & 3565.228266 & 3593.838664 & $1 / 7 / 2010$ \\
3646.175733 & 3578.974564 & 3600.70516 & $1 / 8 / 2010$ \\
3682.757122 & 3609.94033 & 3644.232887 & $1 / 11 / 2010$ \\
3666.216319 & 3599.600665 & 3632.189905 & $1 / 12 / 2010$ \\
3673.069508 & 3604.710616 & 3638.949944 & $1 / 13 / 2010$ \\
3674.014316 & 3621.397797 & 3643.474379 & $1 / 14 / 2010$ \\
3662.450392 & 3588.728714 & 3644.232887 & $1 / 15 / 2010$ \\
3670.873826 & 3604.045258 & 3618.89605 & $1 / 19 / 2010$ \\
3637.273241 & 3565.281494 & 3620.333224 & $1 / 20 / 2010$ \\
\hline
\end{tabular}




\section{Continued}

\begin{tabular}{|c|c|c|c|}
\hline 3642.982014 & 3525.080557 & 3614.318387 & $1 / 21 / 2010$ \\
\hline 3564.230228 & 3462.257445 & 3534.834707 & $1 / 22 / 2010$ \\
\hline 3524.468428 & 3457.879389 & 3503.017283 & $1 / 25 / 2010$ \\
\hline 3525.439851 & 3452.117387 & 3475.937208 & $1 / 26 / 2010$ \\
\hline 3514.381599 & 3433.726889 & 3475.058935 & $1 / 27 / 2010$ \\
\hline 3511.666938 & 3414.68434 & 3499.903407 & $1 / 28 / 2010$ \\
\hline 3490.907765 & 3403.985382 & 3454.898584 & $1 / 29 / 2010$ \\
\hline 3474.673027 & 3415.496077 & 3436.308479 & $2 / 1 / 2010$ \\
\hline 3508.180461 & 3437.23998 & 3472.051516 & $2 / 2 / 2010$ \\
\hline 3501.393809 & 3437.213366 & 3466.635501 & $2 / 3 / 2010$ \\
\hline 3452.569831 & 3365.421226 & 3437.838802 & $2 / 4 / 2010$ \\
\hline 3401.32395 & 3306.590262 & 3370.690862 & $2 / 5 / 2010$ \\
\hline 3395.375648 & 3328.706766 & 3368.189116 & $2 / 8 / 2010$ \\
\hline 3430.293641 & 3356.172748 & 3379.433668 & $2 / 9 / 2010$ \\
\hline 3411.051485 & 3348.121915 & 3386.712685 & $2 / 10 / 2010$ \\
\hline 3434.37894 & 3354.881954 & 3375.627819 & $2 / 11 / 2010$ \\
\hline 3418.237353 & 3358.847488 & 3387.085286 & $2 / 12 / 2010$ \\
\hline 3477.600427 & 3413.573036 & 3434.350499 & $2 / 16 / 2010$ \\
\hline 3499.23906 & 3445.969474 & 3481.796927 & $2 / 17 / 2010$ \\
\hline 3506.306131 & 3452.599125 & 3473.226224 & $2 / 18 / 2010$ \\
\hline 3535.749983 & 3472.788804 & 3484.011367 & $2 / 19 / 2010$ \\
\hline 3547.450975 & 3496.559861 & 3527.931096 & $2 / 22 / 2010$ \\
\hline 3535.476595 & 3467.061332 & 3510.420616 & $2 / 23 / 2010$ \\
\hline 3529.298034 & 3473.567959 & 3498.036155 & $2 / 24 / 2010$ \\
\hline 3509.791824 & 3433.626021 & 3458.777686 & $2 / 25 / 2010$ \\
\hline 3530.528278 & 3472.87082 & 3507.098956 & $2 / 26 / 2010$ \\
\hline 3558.263457 & 3503.695279 & 3521.684187 & $3 / 1 / 2010$ \\
\hline 3569.513359 & 3518.567568 & 3549.829447 & $3 / 2 / 2010$ \\
\hline 3567.996058 & 3514.562439 & 3540.46592 & $3 / 3 / 2010$ \\
\hline 3561.73548 & 3514.234374 & 3537.280954 & $3 / 4 / 2010$ \\
\hline 3592.655625 & 3543.842257 & 3560.054146 & $3 / 5 / 2010$ \\
\hline 3608.648803 & 3560.928986 & 3583.989236 & $3 / 8 / 2010$ \\
\hline 3621.101611 & 3561.448423 & 3576.197687 & $3 / 9 / 2010$ \\
\hline 3632.501877 & 3577.25023 & 3598.478782 & $3 / 10 / 2010$ \\
\hline 3629.207555 & 3576.908495 & 3598.847855 & $3 / 11 / 2010$ \\
\hline 3645.815856 & 3595.608211 & 3631.599697 & $3 / 12 / 2010$ \\
\hline 3642.61722 & 3592.5326 & 3619.037534 & $3 / 15 / 2010$ \\
\hline 3665.88251 & 3615.661197 & 3637.696242 & $3 / 16 / 2010$ \\
\hline 3690.897481 & 3640.990563 & 3662.834238 & $3 / 17 / 2010$ \\
\hline 3701.299882 & 3640.826531 & 3662.178107 & $3 / 18 / 2010$ \\
\hline
\end{tabular}

